\title{
Solution of nonlinear Cauchy problem for hyperelastic solids
}

\author{
S Andrieux ${ }^{1}$ and $\mathbf{T} \mathbf{N}$ Baranger ${ }^{2,3}$ \\ ${ }^{1}$ ONERA, Chemin de la Hunière, BP 80100, F-92123 Palaiseau, France \\ ${ }^{2}$ Université de Lyon, CNRS, LMC2-Université Lyon1, F-69622 Villeurbanne, France \\ E-mail: stephane.andrieux@onera.fr and thouraya.baranger@univ-lyon1.fr
}

\begin{abstract}
The problem of expanding given (measured) fields at the surface of a solid within the solid and up to inaccessible parts of its boundary is addressed for a nonlinear hyperelastic medium. The problem is formulated as a nonlinear Cauchy problem and is solved thanks to a technique consisting of splitting the unknown field into two solutions of well posed problems and minimizing a specially designed error in constitutive equation between the two fields, taking advantage of the convexity of the hyperelastic potential. The minimization involves as unknowns the boundary conditions fields on the inaccessible part of the boundary of the solid. Two illustrations are given, the first one with a twice-differentiable hyperelastic potential describing a material with nonlinear compressibility, the second one deals with a geomaterial with asymmetric elasticity in the tension and compression ranges, and involves an only one-differentiable potential.
\end{abstract}

Keywords: Cauchy problem, boundary data identification, hyperelasticity, convexity, data completion

\section{The nonlinear elliptic Cauchy problem for hyperelastic solids}

The applications aimed at here are the usual applications of Cauchy problems encountered in mechanics such as identification of missing boundary conditions, identification of mechanical quantities inside the solid such as surface tractions on an interface or internal pressure in cavities [1], determination of fracture mechanics parameters for cracked bodies [2], identification of contact surfaces and friction coefficient [1]. The solution of the Cauchy problem can also be used as a first step for geometrical inverse problems solution [3]. The new aspects

3 Author to whom any correspondence should be addressed. 
addressed here lie in the constitutive equation of the material which is no more considered as linearly elastic as in preceding papers $[1,4,5]$ but rather described via an energy density potential, the hyperelastic potential or the free energy, which is no longer quadratic. Nevertheless the framework of linearized strain theory is kept and no energy dissipation is assumed to occur in the strain process.

Hyperelastic potentials are used for the modelling of materials such as geomaterials like concrete or rocks where the nonlinearity arises from the asymmetric behaviour of these cracked media in the tension or compression range (with piecewise quadratic potential) or such as elastomeric materials (with non-quadratic regular potential). They can also be used for elastoplastic materials when the assumptions of radial and monotonic strain paths are valid everywhere within the solid and for the whole loading sequence.

Let $\Omega$ be a domain in $R^{n}, n=2$ or 3 , with piecewise Lipschitz boundary $\partial \Omega=\bar{\Gamma}_{m} \cup \bar{\Gamma}_{u}$. Where $\Gamma_{m}$ and $\Gamma_{u}$ are two open disjoint parts of $\partial \Omega$, with outward unit normal $\mathbf{n}$. The family of Cauchy problems addressed here can be stated as follows. Provided the Cauchy data $\left(U_{m}, T_{m}\right)$ on $\Gamma_{m}$ are given, find a $n$ components vector field $u$ in $R^{n}$ such that:

$$
\nabla \cdot \sigma=0, \quad \sigma=\frac{\partial \varphi(\varepsilon)}{\partial \varepsilon}, \quad \varepsilon=\frac{1}{2}\left(\nabla u+{ }^{t} \nabla u\right) \quad \text { in } \Omega,
$$

and

$$
u=U_{m} \quad \text { and } \quad \sigma \cdot \mathbf{n}=\mathrm{T}_{m} \quad \text { on } \Gamma_{m} .
$$

These equations are respectively the mechanical equilibrium equation, the constitutive equation and the compatibility equation. The potential $\varphi$ is a convex, lower semi-continuous (lsc) differentiable real function, $\sigma$ is the (second order) Cauchy stress tensor and $\varepsilon$ is the (second order) linearized strain tensor, both being symmetric. If the potential $\varphi$ is not quadratic, the material is no longer linearly elastic, and the second order operator on $u$ defined by (1) turns out to be nonlinear. The associated Cauchy problem is then a nonlinear elliptic one.

Linear elliptic Cauchy problems have been extensively studied since the 1920s [6] and for operator with analytical coefficients, uniqueness of solution is ensured [7,8] although compatibility conditions between the Cauchy data have to be satisfied [9]. Theoretical results for existence and data compatibility conditions for nonlinear elliptic Cauchy problems have been addressed by [10]. Numerous numerical approaches are available in the literature for linear elliptic Cauchy problems, although the complexity of the algorithms and the large amount of computation needed restrain the applications in almost all the papers to twodimensional problems: fixed point algorithms [11-13], variational approaches based on Steklov-Poincaré operators [14-16], least-squares method with vanishing regularization [17], methods using fundamental solutions [13, 18] or boundary integral techniques [19], moment methods associated to Backus-Gilbert techniques [20], quasi-reversibility methods [21, 22], and energy error based methods [1, 4, 5, 16, 23, 24]. Recently, Carleman estimates have been used to derive convex, Thikonov regularized, functionals, the minimization of which is used for the solution of linear and quasilinear Cauchy problems and some inverse problems [2527]. This paper extends the energy error methods derived by the authors to the above class of nonlinear problems.

As noted by [28], the Cauchy problem for special nonlinear scalar elliptic equations can be reduced to the Cauchy problem for linear ones by a change of the unknown function. Namely, if we consider the following class of mixed nonlinear boundary value problems where $u$ is a scalar function: 


$$
\begin{aligned}
& \nabla \cdot(q(u) \nabla u)=0 \quad \text { in } \quad \Omega, \\
& u=T \quad \text { on } \quad \Gamma_{1}, \\
& q(u) \nabla u \cdot \mathbf{n}=\phi \quad \text { on } \quad \Gamma_{2}
\end{aligned}
$$

with $\partial \Omega=\bar{\Gamma}_{1} \cup \bar{\Gamma}_{2}$. Then provided that $q$ is a regular $\left(C^{\infty}\right)$ and bounded function $\left(q(t) \in\left[q_{\min }, q_{\max }\right], \quad 0<q_{\min } \leqslant q_{\max }<\infty\right)$, the following primitive function $Q$ of $q$ can be defined, and is a monotonic, then an invertible function:

$$
Q(s)=\int_{0}^{s} q(t) \mathrm{d} t .
$$

The function $U:=Q(u)$ satisfies the following linear problem:

$$
\begin{aligned}
& \Delta U=0 \quad \text { in } \quad \Omega, \\
& U=Q(T) \quad \text { on } \quad \Gamma_{1}, \\
& \nabla U \cdot \mathbf{n}=\phi \quad \text { on } \quad \Gamma_{2}
\end{aligned}
$$

and solving $(3 a)-(3 c)$ for $u$ is equivalent to solving $(5 a)-(5 c)$ for $U$ and computing $u=Q^{-1}(U)$.

Unfortunately this method cannot be used for the general nonlinear operators arising for hyperelastic media where firstly the unknown (the displacement field) is a three components vector field and secondly the nonlinearity is expressed through nonlinear functions of the strain tensor and not of the displacement field itself. Furthermore, the assumption that $q$ does not depends on the space variable $x$ is not relevant for heterogeneous media.

The paper is organized as follows. First, the solution method is derived and the variational form of the data completion problem is detailed. Then the case of incompressible materials is addressed. The next part is devoted to the computation of the gradient of the error in constitutive equation functional. Lastly, two illustrations are displayed.

\section{Solution of the Cauchy problem for hyperelastic solids by constitutive equation error minimization}

To derive a general solution method for problem (1) and (2), the main idea is to seek for the boundary conditions on $\Gamma_{u}$ where no data are available. We proceed with two steps as for the derivation of the energy error method for linear elliptic Cauchy problems [1, 5, 16, 23]. The first step is exactly similar: two well-posed direct problems are defined, the solutions of which are denoted by $u_{1}$ and $u_{2}$. These problems are parametrized by two fields, respectively by $\eta$ (a natural boundary condition quantity, that is: a surface traction field) and $\tau$ (a Dirichlet boundary condition, that is: a prescribed displacement field), defined on $\Gamma_{u}$, namely:

$$
\begin{aligned}
& \nabla \cdot \sigma_{1}=0, \quad \sigma_{1}=\frac{\partial \varphi\left(\varepsilon\left(u_{1}\right)\right)}{\partial \varepsilon} \text { in } \Omega, \\
& u_{1}=U_{m} \quad \text { on } \quad \Gamma_{m}, \\
& \sigma_{1} \cdot \mathbf{n}=\eta \quad \text { on } \quad \Gamma_{u}
\end{aligned}
$$

and

$$
\nabla \cdot \sigma_{2}=0, \quad \sigma_{2}=\frac{\partial \varphi\left(\varepsilon\left(u_{2}\right)\right)}{\partial \varepsilon} \quad \text { in } \quad \Omega,
$$




$$
\begin{array}{lll}
\sigma_{2} \cdot \mathbf{n}=T_{m} & \text { on } \quad \Gamma_{m}, \\
u_{2}=\tau \quad \text { on } \quad & \Gamma_{u} .
\end{array}
$$

Because if the solutions of these two problems are equal, then the common solution field $u=u_{1}=u_{2}$ is obviously a solution of the Cauchy problem (1) and (2), the second step of the method is to derive a functional 'measuring the gap' between the two vector fields $u_{1}$ and $u_{2}$, as a functional $E(\eta, \tau)$ of the unknowns $\eta$ and $\tau$. Then the solution method will be to minimize this functional

$$
\left(T_{u}, U_{u}\right)=\arg \min _{(\eta, \tau)} E(\eta, \tau), \quad u=u_{1}\left(T_{u}\right) .
$$

For linear symmetric elliptic operators such as the scalar conduction operator encountered in stationary conduction problems (thermal or electric conduction), or as the Lamé operator arising in linear elasticity problems, a semi-norm of the error has been proposed by the authors by taking the energy of the difference field $u_{1}-u_{2}$. By 'energy', it must be understood the functional appearing as a potential of the system of equations, which are usually and for such symmetric operators, characterized by a weak formulation involving a symmetric bilinear form and Hilbert space $V$, see [5]:

$$
a(u, v)=l(v) \quad \forall v \in V \quad \text { or } \quad u=\arg \min _{V} \frac{1}{2} a(v, v)-l(v) .
$$

In such a case, the 'error in energy' functional is simply:

$$
E(\eta, \tau)=a\left(u_{1}(\eta)-u_{2}(\tau), u_{1}(\eta)-u_{2}(\tau)\right) .
$$

This functional is positive, quadratic, then convex, and achieves its minimum when $u_{1}$ equals $u_{2}$, up to a scalar constant (Laplace operator) or up to a rigid body displacement field (Lamé operator). Furthermore, it can be reduced to an equivalent expression involving only integration over the boundary of the domain. Advantage can be taken of this last feature from the computational standpoint: firstly the evaluation of the functional is very cheap and secondly the gradient of the functional can be computed by adjoint techniques leading to adjoint problems involving only boundary terms. The solution procedure when combined with ad hoc optimization algorithms turns out to be very efficient and allowed to deal with complex threedimensional applications [2-4]. This choice has also been used for the resolution of Cauchy problems in aquifers by [24].

To derive error functionals with similar desirable properties for nonlinear elliptic Cauchy problems (1) and (2), a different way must be followed and advantage has to be taken of the convexity of the potential $\varphi$. The error will no more be an 'error in energy' but rather an 'error in constitutive equation'. The following lemma is the key-point of the building of the new error functional.

Lemma 1. Fenchel inequality or conjugacy formula [29]. Let $\psi$ be a lsc, convex function from $R^{n}$ into $\bar{R}$, then for any pair $(x, y) \in R^{n} \times R^{n}$ :

(i) $\psi(x)+\psi^{*}(y)-x \cdot y \geqslant 0$

(ii) $\psi(x)+\psi^{*}(y)-x \cdot y=0 \Leftrightarrow y \in \partial \psi(x) \Leftrightarrow x \in \partial \psi^{*}(y)$ where $\partial \psi(t)$ is the subdifferential set of $\psi$ at $t$ :

$$
\partial \psi(t)=\left\{g \in R^{n} \text { s.t. } \quad \psi(t)-\psi(z) \geqslant g \cdot(t-z) \quad \forall z \in R^{n}\right\}
$$


and $\psi^{*}$ is the Legendre-Fenchel conjugate function of $\psi$ :

$$
\psi^{*}(y)=\sup _{x}[x \cdot y-\psi(x)] .
$$

The error in constitutive equation for the hyperelastic material with convex potential $\varphi$ is then defined for a pair $(\sigma, \varepsilon)$ by the scalar function $e_{\varphi}$ :

$$
e_{\varphi}(\sigma, \varepsilon) \equiv \varphi(\varepsilon)+\varphi^{*}(\sigma)-\sigma: \varepsilon
$$

and enjoys then the following properties:

$$
\begin{aligned}
& e_{\varphi}(\sigma, \varepsilon) \geqslant 0 \quad \forall(\sigma, \varepsilon), \\
& e_{\varphi}(\sigma, \varepsilon)=0 \Leftrightarrow \sigma \in \partial \varphi(\varepsilon) \Leftrightarrow \varepsilon \in \partial \varphi^{*}(\sigma) .
\end{aligned}
$$

We then define the (positive) error in constitutive equation for the two fields $u_{1}$ and $u_{2}$ solutions of $(6 a)-(6 c)$ and $(7 a)-(7 c)$ as:

$$
\operatorname{ECE}\left(u_{1}, u_{2}\right)=\int_{\Omega}\left[e_{\varphi}\left(\sigma_{1}, \varepsilon\left(u_{2}\right)\right)+e_{\varphi}\left(\sigma_{2}, \varepsilon\left(u_{1}\right)\right] \mathrm{d} \Omega .\right.
$$

This functional is positive and its minimum is zero, with the following characterization and relation with the solution of the Cauchy problem of the corresponding fields $u_{1}$ and $u_{2}$.

Theorem 1. If the potential $\varphi$ is lsc, strictly convex and differentiable, then the following equivalence holds for $u_{1}$ and $u_{2}$ satisfying $(6 a)-(6 c)$ and $(7 a)-(7 c)$ :

$\operatorname{ECE}\left(u_{1}, u_{2}\right)=0 \Leftrightarrow \exists(t, \omega) \in R^{3} \times R^{3} \mid u_{2}(x)=u_{1}(x)+t+\omega \wedge x$.

Consequently, $u_{1}$ solves the Cauchy problem (1) and (2)

Proof. Note first that because of the differentiability of $\varphi$, the subdifferential $\partial \varphi(\varepsilon)$ reduces at every point $\varepsilon$ to the set $\left\{\frac{\partial \varphi}{\partial \varepsilon}\right\}$, the same property holds for $\varphi^{*}$. The implication leading to $\mathrm{ECE}=0$ is then obvious because if $u_{1}$ and $u_{2}$ differ from a rigid body displacement field $t+\omega \wedge x$, then $\varepsilon\left(u_{1}\right)=\varepsilon\left(u_{2}\right)$ a.e. in $\Omega$, so that by the constitutive equation we get $\sigma_{1}=\sigma_{2}$. The field $u_{1}$ is then a solution of (1) and (2) and furthermore $\operatorname{ECE}\left(u_{1}, u_{2}\right)$ is zero. The other implication is straightforward too. If ECE is null for a couple of fields $\left(u_{1}, u_{2}\right)$, then the two positive errors $e_{\varphi}\left(\sigma_{2}, \varepsilon\left(u_{1}\right)\right)$ and $e_{\varphi}\left(\sigma_{1}, \varepsilon\left(u_{2}\right)\right)$ vanish simultaneously a.e. in the domain $\Omega$. Using lemma 1 for $\left(\sigma_{2}, \varepsilon\left(u_{1}\right)\right.$ and the constitutive equation for $\left(\sigma_{2}, \varepsilon\left(u_{2}\right)\right.$, we have then:

$$
\left.\begin{array}{l}
\varepsilon\left(u_{1}\right)=\frac{\left.\partial \varphi^{*}\left(\sigma_{2}\right)\right)}{\partial \sigma} \\
\varepsilon\left(u_{2}\right)=\frac{\left.\partial \varphi^{*}\left(\sigma_{2}\right)\right)}{\partial \sigma}
\end{array}\right\} \Leftrightarrow \varepsilon\left(u_{1}\right)=\varepsilon\left(u_{2}\right) \quad \text { a.e. in } \Omega .
$$

So that $u_{1}$ and $u_{2}$ differ only by a rigid body displacement field. The surface traction fields $\sigma_{1} \cdot \mathbf{n}$ and $\sigma_{2} \cdot \mathbf{n}$ are then equal on $\Gamma_{m}$ and $u_{1}$ solves the Cauchy problem (1) and (2).

Taking advantage of theorem 1 and using as unknowns the Neumann and Dirichlet fields $\eta$ and $\tau$ on $\Gamma_{u}$, we can recast the Cauchy problem into the following optimization problem: 


$$
\begin{aligned}
& \left(T_{u}, U_{u}\right)=\arg \min _{\eta, \tau} E(\eta, \tau), \quad E(\eta, \tau)=\operatorname{ECE}\left(u_{1}(\eta), u_{2}(\tau)\right), \\
& (\eta, \tau) \in H^{-1 / 2}\left(\Gamma_{u}\right)^{n} \times H^{1 / 2}\left(\Gamma_{u}\right)^{n}, \\
& \left(u_{1}(\eta), u_{2}(\tau)\right) \text { given by }(6 a)-(7 c) .
\end{aligned}
$$

where, $\left(u_{1}, u_{2}\right) \in H^{1}(\Omega)^{n} \times H^{1}(\Omega)^{n}$. $H^{1 / 2}\left(\Gamma_{u}\right)$ is the space of restrictions to $\Gamma_{u}$ of the functions of $H^{1 / 2}(\Omega)=\operatorname{tr}\left(H^{1}(\Omega)\right)$ and $H^{-1 / 2}\left(\Gamma_{u}\right)$ is the topological dual of $H_{00}^{1 / 2}\left(\Gamma_{u}\right)$, $H^{-1 / 2}\left(\Gamma_{u}\right)=\left(H_{00}^{1 / 2}\left(\Gamma_{u}\right)\right)^{\prime}$.

This formulation is similar to the error in energy approach used by the authors for linear elliptic Cauchy problems except apparently for the form of the error which is no more the energy of the difference field $u_{1}-u_{2}$. In fact, for linear problems, i.e. problems where the constitutive potential $\varphi$ is quadratic, the following proposition shows that the current formulation via an error in constitutive equation encompasses the error in energy.

Proposition 1. For any pairs $\left(\sigma_{1}, \varepsilon_{1}\right)$ and $\left(\sigma_{2}, \varepsilon_{2}\right)$ satisfying the constitutive equation for the potential $\varphi$, one have:

$$
e_{\varphi}\left(\sigma_{1}, \varepsilon_{2}\right)+e_{\varphi}\left(\sigma_{2}, \varepsilon_{1}\right)=\left(\sigma_{1}-\sigma_{2}\right):\left(\varepsilon_{1}-\varepsilon_{2}\right) .
$$

Proof. The property follows from the definition of the error $e_{\varphi}$ and the conjugacy formula (16) for the two pairs $\left(\sigma_{1}, \varepsilon_{1}\right)$ and $\left(\sigma_{2}, \varepsilon_{2}\right)$ :

$$
\begin{aligned}
& e_{\varphi}\left(\sigma_{1}, \varepsilon_{2}\right)=\varphi\left(\varepsilon_{2}\right)+\varphi^{*}\left(\sigma_{1}\right)-\sigma_{1}: \varepsilon_{2}, \\
& e_{\varphi}\left(\sigma_{2}, \varepsilon_{1}\right)=\varphi\left(\varepsilon_{1}\right)+\varphi^{*}\left(\sigma_{1}\right)-\sigma_{2}: \varepsilon_{1}, \\
& 0=\varphi\left(\varepsilon_{1}\right)+\varphi^{*}\left(\sigma_{1}\right)-\sigma_{1}: \varepsilon_{1}, \\
& 0=\varphi\left(\varepsilon_{2}\right)+\varphi^{*}\left(\sigma_{2}\right)-\sigma_{2}: \varepsilon_{2}
\end{aligned}
$$

by substracting the two last equalities to the sum of the two first equalities.

Corollary 1. If the potential $\varphi$ is quadratic, then the error in constitutive equation $\operatorname{ECE}\left(u_{1}, u_{2}\right)$ is exactly twice the energy of the difference of the fields $u_{1}$ and $u_{2}$ :

$$
\varphi(\varepsilon) \quad \text { quadratic } \quad \Rightarrow \operatorname{ECE}\left(u_{1}, u_{2}\right)=2 \int_{\Omega} \varphi\left(\varepsilon\left(u_{1}-u_{2}\right)\right) \mathrm{d} \Omega .
$$

Proof. The proof relies on the proposition 1 . If the potential $\varphi$ is quadratic, the identity (22) reduces to the result:

$$
\begin{aligned}
& \boldsymbol{e}_{\varphi}\left(\sigma_{1}, \varepsilon_{2}\right)+\boldsymbol{e}_{\varphi}\left(\sigma_{2}, \varepsilon_{1}\right)=\left(\sigma_{1}-\sigma_{2}\right):\left(\varepsilon_{1}-\varepsilon_{2}\right), \\
& =\left(\frac{\partial \varphi}{\partial \varepsilon}\left(\varepsilon_{1}\right)-\frac{\partial \varphi}{\partial \varepsilon}\left(\varepsilon_{2}\right)\right):\left(\varepsilon_{1}-\varepsilon_{2}\right), \\
& =2 \varphi\left(\varepsilon\left(u_{1}-u_{2}\right)\right)
\end{aligned}
$$

and the implication (27) is then established. 
Remark 1. Interestingly, the proposition 1 derives a form (22) of the error in constitutive equation that can also be obtained with reference to the concept of Bregman distance introduced in the context of convex optimization [30, 31]. Let $J$ be a convex function, the generalized Bregman distance between $u$ and $v$ with respect to $J$ is the non-negative scalar:

$$
D_{J}^{p}(u, v)=J(u)-J(v)-(p, u-v) \quad \text { for } \quad p \in \partial J(v) .
$$

It is readily seen that the error in constitutive equation is exactly the symmetrized generalized Bregman distance between $\varepsilon_{1}$ and $\varepsilon_{2}$ with respect to the hyperelastic potential $\varphi$ for any pairs $\left(\sigma_{1}, \varepsilon_{1}\right)$ and $\left(\sigma_{2}, \varepsilon_{2}\right)$ satisfying the constitutive equation:

$$
\boldsymbol{e}_{\varphi}\left(\sigma_{1}, \varepsilon_{2}\right)+\boldsymbol{e}_{\varphi}\left(\sigma_{2}, \varepsilon_{1}\right)=D_{\varphi}^{\sigma_{1}}\left(\varepsilon_{2}, \varepsilon_{1}\right)+D_{\varphi}^{\sigma_{2}}\left(\varepsilon_{1}, \varepsilon_{2}\right)
$$

The proposition 1 have also an important consequence: the energy error in constitutive equation can be calculated without any need of the knowledge, or the computation by the definition (12), of the conjugate potential $\varphi^{*}$ :

$$
\operatorname{ECE}\left(u_{1}, u_{2}\right)=\int_{\Omega}\left(\sigma_{1}-\sigma_{2}\right):\left(\varepsilon_{1}-\varepsilon_{2}\right) \mathrm{d} \Omega .
$$

Furthermore, this expression leads to another alternative equivalent form of the functional $E$ involving only boundary terms.

Proposition 2. The functional E can be computed by the following expression involving only boundary terms:

$$
\begin{aligned}
\mathfrak{E}(\eta, \tau)= & \int_{\Gamma_{m}}\left(\sigma_{1}(\eta) \cdot \mathbf{n}-T_{m}\right) \cdot\left(U_{m}-u_{2}(\tau)\right) \mathrm{d} S \\
& +\int_{\Gamma_{u}}\left(\eta-\sigma_{2}(\tau) \cdot \mathbf{n}\right) \cdot\left(u_{1}(\eta)-\tau\right) \mathrm{d} S .
\end{aligned}
$$

This expression will be used in the numerical applications because of its efficiency from a computational standpoint.

Proof. Using the expression (33), the result (34) is simply obtained by taking into account the definition of the fields $u_{i}$ and using each one as a virtual field in the principle of virtual power expressed for the other one.

Notice that the potential $\varphi$ does not appear any more in the expression of the functional $E$, but the positivity of the scalar product $\left(\sigma_{1}-\sigma_{2}\right):\left(\varepsilon\left(u_{1}\right)-\varepsilon\left(u_{2}\right)\right)$, and the results deduced when the scalar product is zero, are directly grounded on the existence of a convex lsc potential. The general energy error approach developed here can be linked with pioneering works, such as those of [32] who identified distributed parameters in an elliptical equation $[32,33]$, in a similar framework, and more generally with the development of constitutive equation errors for various applications ([34-36]). For identification problems with a nonlinear state equation, [37] used the Fenchel inequality in a penalized least-square method in order to build the penalization of the convex constitutive equation. For the general problem of identification of material parameters for a dissipative medium which behaviour is modelled in the generalized standard materials framework [38], with two potentials (the Helmholtz free energy, similar to the $\varphi$ potential used here, and a dissipation pseudo-potential), see [39, 40]. Remark finally that the choice of mixed problems for the definition of the solutions $\left(u_{1}, u_{2}\right)$ avoid to take into account the compatibility constraints placed on the unknowns $(\eta, \tau)$, 
namely, in the general case (global equilibrium constraint):

$$
\begin{aligned}
& \int_{\Gamma_{m}} T_{m} \mathrm{~d} S+\int_{\Gamma_{u}} \eta \mathrm{d} S=0, \\
& \int_{\Gamma_{m}} T_{m} \wedge O M \mathrm{~d} S+\int_{\Gamma_{u}} \eta \wedge O M \mathrm{~d} S=0
\end{aligned}
$$

and the supplementary constraint for incompressible materials:

$$
\int_{\Gamma_{m}} U_{m} \cdot \mathbf{n} \mathrm{d} S+\int_{\Gamma_{u}} \tau \cdot \mathbf{n} \mathrm{d} S=0
$$

This feature facilitates the minimization procedure which turns out to be an unconstrained optimization problem.

\section{Incompressible materials}

An interesting case in the applications is the case of incompressible materials. In the small perturbations framework, this is modelled by a divergence-free constraint on the displacement field, and the modification of the stress-strain relation where the mean stress or internal pressure appears as a Lagrange multiplier of the preceding constraint and is no more (locally) linked to the strain field via the hyperelastic potential:

$$
\begin{gathered}
\sigma=-p \mathbf{I}+\tilde{\sigma} \equiv-p \mathbf{I}+\frac{\partial \varphi(\tilde{\varepsilon}(u))}{\partial \tilde{\varepsilon}}, \nabla \cdot u=\operatorname{tr}(\varepsilon(u))=0 \quad \text { in } \quad \Omega, \\
\tilde{\varepsilon}=\varepsilon-\frac{1}{3} \operatorname{tr}(\varepsilon) \mathbf{I}, \quad \tilde{\sigma}=\sigma-\frac{1}{3} \operatorname{tr}(\sigma) \mathbf{I} .
\end{gathered}
$$

The Cauchy problem is then slightly modified and consists in finding the displacement field $u$ and the pressure field $p$ such that:

$$
\begin{aligned}
& \nabla \cdot \sigma=0, \quad \nabla \cdot u=0, \quad \sigma=-p \mathbf{I}+\frac{\partial \varphi(\tilde{\varepsilon}(u))}{\partial \tilde{\varepsilon}} \quad \text { in } \Omega, \\
& u=U_{m}, \quad \text { and } \quad \sigma \cdot \mathbf{n}=T_{m} \quad \text { on } \quad \Gamma_{m} .
\end{aligned}
$$

The splitting of the fields is then:

$$
\begin{array}{ll}
\nabla \cdot \sigma_{1}=0, & \nabla \cdot u_{1}=0, \quad \sigma_{1}=-p_{1} \mathbf{I}+\frac{\partial \varphi\left(\tilde{\varepsilon}\left(u_{1}\right)\right)}{\partial \tilde{\varepsilon}} \quad \text { in } \Omega, \\
u_{1}=U_{m} \quad \text { on } \quad \Gamma_{m}, \\
\sigma_{1} \cdot \mathbf{n}=\eta \quad \text { on } \Gamma_{u}
\end{array}
$$

and:

$$
\begin{gathered}
\nabla \cdot \sigma_{2}=0, \quad \nabla \cdot u_{2}=0, \quad \sigma_{2}=-p_{2} \mathbf{I}+\frac{\partial \varphi\left(\tilde{\varepsilon}\left(u_{2}\right)\right)}{\partial \tilde{\varepsilon}} \quad \text { in } \Omega, \\
\sigma_{2} \cdot \mathbf{n}=T_{m} \quad \text { on } \quad \Gamma_{m}, \\
u_{2}=\tau \quad \text { on } \Gamma_{u} .
\end{gathered}
$$

In the error in constitutive equation approach derived here, the Fenchel inequality to be exploited would naturally be related to the hyperelastic potential $\varphi$ which is function of the 
deviatoric part of the strain tensor only, and to use the scalar product between the deviatoric parts of the stress and strain tensors:

$$
\left(\tilde{\sigma}_{1}-\tilde{\sigma}_{2}\right):\left(\tilde{\varepsilon}_{1}-\tilde{\varepsilon}_{2}\right) .
$$

But in order to obtain the equivalent boundary expression of the error functional, it is preferred to use an equivalent expression, when applied to pairs $\left(u_{1}, u_{2}\right)$ solutions of problems (42)-(47):

$\left(\tilde{\sigma}_{1}-\tilde{\sigma}_{2}\right):\left(\tilde{\varepsilon}_{1}-\tilde{\varepsilon}_{2}\right)-\left(p_{1}-p_{2}\right)\left(\operatorname{tr}\left(\varepsilon_{1}\right)-\operatorname{tr}\left(\varepsilon_{2}\right)\right)=\left(\sigma_{1}-\sigma_{2}\right):\left(\varepsilon_{1}-\varepsilon_{2}\right)$.

The functional $E$ has exactly the same boundary expression form than in the compressible case (34), only the definition of the problems for the fields $u_{1}$ and $u_{2}$ is modified. The theorem 1 is also slightly modified.

Theorem 2. If the potential $\varphi$ is lsc, strictly convex and differentiable, then the following equivalence holds for $u_{1}$ and $u_{2}$ satisfying (42)-(44) and (45)-(47):

$\operatorname{ECE}\left(u_{1}, u_{2}\right)=0 \Leftrightarrow \exists(t, \omega) \in R^{3} \times R^{3} \mid u_{2}(x)=u_{1}(x)+t+\omega \wedge x$.

Consequently, $\left(u_{1}, p_{2}\right)$ solve the Cauchy problem (40) and (41).

\section{Computation of the gradient}

The variational formulation of the nonlinear elliptic Cauchy problem derived in the preceding parts necessitates using minimization algorithms. Due to ill-posedness of the Cauchy problem and for efficiency of the method, it is important to use robust and efficient algorithms that take advantage of the knowledge of the gradient of the objective functional $E$. The gradient has to be computed by an adjoint method because of the implicit dependence of the $u_{1}$ and $u_{2}$ fields with respect to the variables $(\eta, \tau)$, and the relatively high cost of evaluation of the function itself $[41,42]$. The following property gives the general expression of the gradient of the functional $E$, for twice-differentiable hyperelastic potentials $\varphi$.

Proposition 3. Provided the hyperelastic potential is twice differentiable, the gradients of the functional $E$ can be computed by the following expression:

$$
\left\{\begin{array}{l}
\nabla_{\eta} E(\eta, \tau)=-\left.v_{1}^{*}\right|_{\Gamma_{u}} \\
\nabla_{\tau} E(\eta, \tau)=\left.\left[\sigma_{2} \cdot \mathbf{n}+\sigma_{2}^{*}\left(v_{2}^{*}\right) \cdot \mathbf{n}-\eta-\mathcal{T}\left[\varepsilon\left(u_{2}\right)\right]: \varepsilon\left(u_{1}-u_{2}\right) \cdot \mathbf{n}\right]\right|_{\Gamma_{u}},
\end{array}\right.
$$

where $v_{1}^{*}$ and $v_{2}^{*}$ are adjoint fields solutions of:

$$
\begin{cases}\nabla \cdot \sigma_{1}^{*}=-\nabla \cdot\left(\mathcal{T}\left[\varepsilon\left(u_{1}\right)\right]: \varepsilon\left(u_{1}-u_{2}\right)\right) & \text { in } \Omega \\ \sigma_{1}^{*}=\mathcal{T}\left[\varepsilon\left(u_{1}\right)\right]: \varepsilon\left(v_{1}^{*}\right) & \text { in } \Omega \\ v_{1}^{*}=0 & \text { on } \Gamma_{m} \\ \sigma_{1}^{*} \cdot \mathbf{n}=-\mathcal{T}\left[\varepsilon\left(u_{1}\right)\right]: \varepsilon\left(u_{1}-u_{2}\right) \cdot \mathbf{n}+\sigma_{2}^{*} \cdot \mathbf{n}-\eta & \text { on } \Gamma_{u}\end{cases}
$$


and:

$$
\begin{cases}\nabla \cdot \sigma_{2}^{*}=\nabla \cdot\left(\mathcal{T}\left[\varepsilon\left(u_{2}\right)\right]: \varepsilon\left(u_{1}-u_{2}\right)\right) & \text { in } \Omega \\ \sigma_{2}^{*}=\mathcal{T}\left[\varepsilon\left(u_{2}\right)\right]: \varepsilon\left(v_{2}^{*}\right) & \text { in } \Omega \\ v_{2}^{*}=0 & \text { on } \Gamma_{u} \\ \sigma_{2}^{*} \cdot \mathbf{n}=\mathcal{T}\left[\varepsilon\left(u_{2}\right)\right]: \varepsilon\left(u_{1}-u_{2}\right) \cdot \mathbf{n}+\sigma_{2}^{*} \cdot \mathbf{n}-T_{m} & \text { on } \Gamma_{m}\end{cases}
$$

the fourth-order tensor $\mathcal{T}$ being the second derivative of the hyperelastic potential $\varphi$ or tangent rigidity tensor:

$$
\mathcal{T}[\omega]=\frac{\partial^{2} \varphi}{\partial \varepsilon^{2}}(\omega) \quad \text { or } \quad(\mathcal{T}[\omega])_{i j k l}=\frac{\partial^{2} \varphi}{\partial \varepsilon_{i j} \partial \varepsilon_{k l}}(\omega) .
$$

Proof. Following the usual Lagrangian method for the computation of the gradient of the ECE functional (34) with state equations $(6 a)-(7 a)$, the following Lagrangian:

$$
\begin{aligned}
& L\left(u_{1}, u_{2}, \lambda_{1}, \lambda_{2}, v_{1}, v_{2} ; \eta, \tau\right)=\operatorname{ECE}\left(u_{1}, u_{2}\right), \\
& +\int_{\Omega}\left[\frac{\partial \varphi\left(\varepsilon\left(u_{1}\right)\right)}{\partial \varepsilon}: \varepsilon\left(v_{1}\right)+\frac{\partial \varphi\left(\varepsilon\left(u_{2}\right)\right)}{\partial \varepsilon}: \varepsilon\left(v_{2}\right)\right] \mathrm{d} \Omega, \\
& -\int_{\Gamma_{m}} T_{m} \cdot v_{2} \mathrm{~d} S-\int_{\Gamma_{u}}\left[\lambda_{1} \cdot\left(u_{2}-\tau\right)+\lambda_{2} \cdot v_{2}+\eta \cdot v_{1}\right] \mathrm{d} S
\end{aligned}
$$

is defined on the following space product where the functional regularity for the fields in the function space $V(\Omega)$ of displacement fields in the domain $\Omega$ and in the space $\Lambda_{1}\left(\Gamma_{u}\right)$ of surface traction fields on $\Gamma_{u}$, is not precisely specified as it falls outside of the scope of this paper:

$$
\begin{gathered}
\left(u_{1}, u_{2}, \lambda_{1}, \lambda_{2}, v_{1}, v_{2}\right) \in U_{1} \times V(\Omega) \times U_{1}^{0} \times V(\Omega) \times \Lambda_{1}\left(\Gamma_{u}\right)^{3} \times \Lambda_{1}\left(\Gamma_{u}\right)^{3} \\
\text { with : } \quad U_{1}=\left\{u \in V(\Omega),\left.u\right|_{\Gamma_{m}}=U_{m}\right\}, \quad U_{0}=\left\{u \in V(\Omega),\left.u\right|_{\Gamma_{m}}=0\right\} .
\end{gathered}
$$

Following the classical approach, the stationarity condition of the Lagrangian with respect to primal fields $u_{1}$ and $u_{2}$ leads to the definition of the two adjoint and well posed problems (52) and (53) where the fourth-order tensor $\mathcal{T}$ is the second derivative of the hyperelastic potential $\varphi$, or tangent rigidity tensor (54). The gradient of the functional $E$ is then obtained by partial derivatives of the Lagrangian with respect to the two control fields $\eta$ and $\tau$.

For linear elasticity, i.e. for quadratic hyperelastic potentials, the tensor $\mathcal{T}$ is constant and reduces to the Hooke tensor. In this case, the adjoint problems defined in [1] for linear elasticity are recovered. They are far more simple that in the truly nonlinear cases, especially because the source terms disappear and only boundary conditions involving the data and surface traction fields associated to $u_{1}$ and $u_{2}$ appear. Nevertheless, for the non-quadratic cases, the adjoint problems remain linearly elastic problems, with a space-dependent rigidity tensor. 


\section{Illustrations}

We illustrate the proposed approach for solving nonlinear Cauchy problems in hyperelasticity with two examples. The first one is a pressurized hollow sphere, with nonlinear compressibility, where, because of the spherical symmetry, the Cauchy data reduce to the pair (external radial displacement, external pressure) and the unknowns $\eta$ and $\tau$ are scalars, respectively the inner pressure and the inner radial displacement component. The second illustration deals with a bidimensional problem, with a non-smooth (i.e. non-twice-differentiable) potential, modelling the asymmetric elasticity in the traction and compression ranges observed for cracked geomaterials.

\subsection{Pressurized sphere with nonlinear compressibility}

Consider the simple case of a homogeneous domain $\Omega$, lying between to concentric spheres with internal radius $a$, and external radius $b$, as shown on figure 1 . We suppose that the situation is fully symmetric so that the stress vector and the prescribed displacement on the spherical boundaries have only radial constant component in the spherical coordinate system centred on the common centre of the two limiting spheres:

$u(a, \varphi, \theta)=U_{a} \mathbf{e}_{r}, u(b, \varphi, \theta)=U_{b} \mathbf{e}_{r}, \quad \sigma(a, \varphi, \theta) \cdot \mathbf{n}=P_{a} \mathbf{e}_{r}, \sigma(b, \varphi, \theta) \cdot \mathbf{n}=P_{b} \mathbf{e}_{r}$,

where $U_{a}, U_{b}, P_{a}$ and $P_{b}$ are four scalars. The boundary where the Cauchy data are given is the external boundary $r=b$, the Cauchy data reduce to the pair of scalars $\left(U_{b}, P_{b}\right)$. The unknowns of the data completion or Cauchy problem reduce also to the pair $\left(U_{a}, P_{a}\right)$ on the internal boundary $r=a$.

The material constituting the hollow sphere is a nonlinear isotropic material with regular (polynomial) potential. A general form of convex potentials for isotropic material can be built using only the two first invariants $I_{1}$ and $I_{2}$ of the strain tensor $\varepsilon$ and a convex function $G$ on $R^{2}$ as follows:

$$
\varphi(\varepsilon)=G\left(I_{1}(\varepsilon), I_{2}(\varepsilon)\right), \quad I_{1}(\varepsilon)=\operatorname{tr}(\varepsilon), \quad I_{2}(\varepsilon)=I_{2}(\tilde{\varepsilon})=\frac{1}{2} \tilde{\varepsilon}: \tilde{\varepsilon} .
$$

Because, unlike the third one, the first two invariants are convex functions of the strain tensor, the potential $\varphi$ is necessarily convex as soon as $G$ enjoys this property. Here the $G$ function is chosen as a polynomial convex function ( $\varphi$ is then lsc):

$$
G(X, Y)=\frac{1}{2} \kappa X^{2}+\frac{1}{4} \alpha \kappa X^{4}+2 \mu Y \quad \kappa, \mu, \alpha>0,
$$

where $X=I_{1}(\varepsilon)$ and $Y=I_{2}(\tilde{\varepsilon})$. The material constants $\kappa$ and $\mu$ can be related to the compressibility and shear or Coulomb moduli encountered in isotropic linear elasticity, whereas the non dimensional coefficient $\alpha$ is a measure of the nonlinearity in the compressibility behaviour of the material.

The figures 2(a) and (b) display the isolines of the potential $\varphi$ in the plane of principal strains in two-dimensions $\left(\varepsilon_{1}, \varepsilon_{2}=\varepsilon_{3}\right)$ for two values of this parameter: $\alpha=0$, i.e. linear elasticity; $\alpha=200$, i.e. nonlinear elasticity. The stress-strain relation is derived by the chain rule and reads:

$$
\sigma=\kappa \operatorname{tr}(\varepsilon) \mathbf{I}+\alpha \kappa \operatorname{tr}(\varepsilon)^{3} \mathbf{I}+2 \mu \tilde{\varepsilon} .
$$

The stress strain law $\left(\sigma_{1}, \varepsilon_{1}\right)$ and $\left(\sigma_{2}, \varepsilon_{1}\right)$ are depicted in figures 3(a) and (b) for $\alpha=200$ and three values of the orthogonal strains $\varepsilon_{2}=\varepsilon_{3}$. The energy error gap reduces to a two-variables function and has a very simple form when using the expression (34) for the ECE functional: 


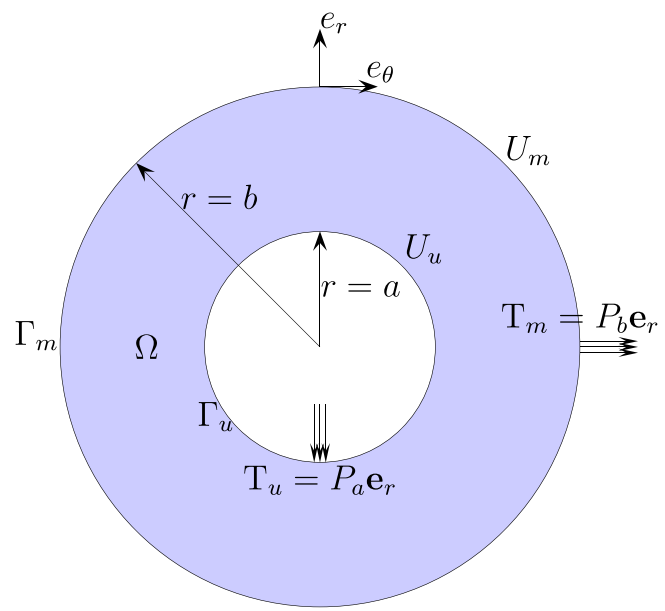

Figure 1. Geometry and notations of the pressurized hollow sphere for the hyperelastic material with nonlinear compressibility.

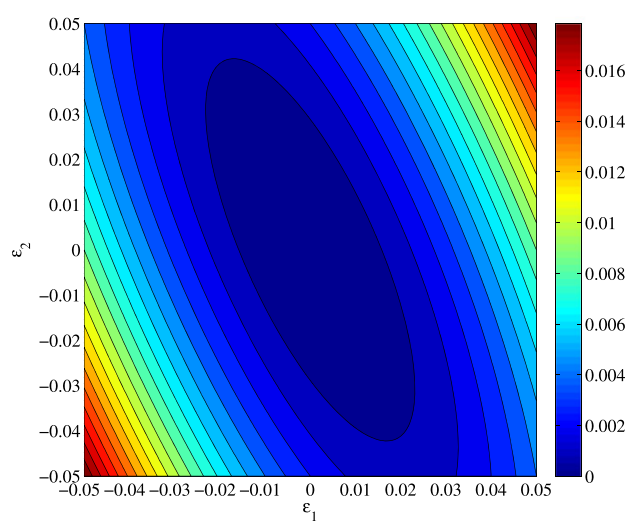

(a)

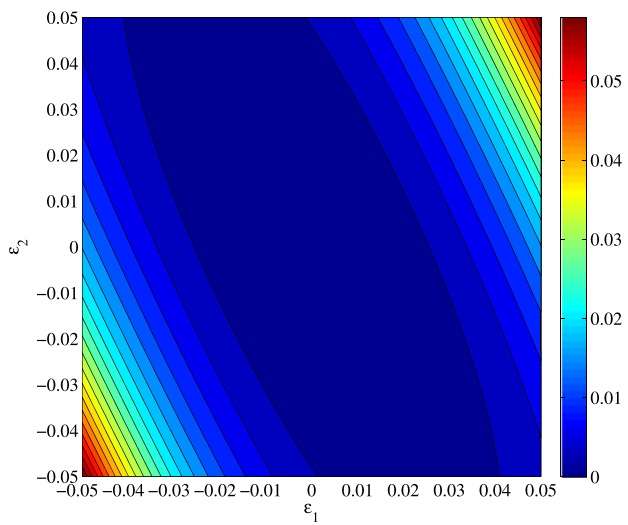

(b)

Figure 2. Isolines of the hyperelastic potential $\varphi$ for $\alpha=0$ (left, linear) and $\alpha=200$ (right, nonlinear).

$$
\begin{aligned}
E(\tau, \eta)= & 4 \pi\left[a^{2}\left(\eta+\sigma_{2}^{r r}(a ; \tau)\right)\left(u_{1}(a ; \eta)-\tau\right)\right. \\
& \left.+b^{2}\left(\sigma_{1}^{r r}(b ; \eta)-p_{b}\right)\left(U_{b}-u_{2}(b ; \tau)\right)\right] .
\end{aligned}
$$

Consider the following material data: $\kappa=Y / 3(1-2 \nu)$ and $\mu=1 / 2(1+\nu)$ obtained from ( $Y=1, \nu=0.4$ ) and $\alpha=200.0$, where $Y$ is the Young modulus and $\nu$ is the Poisson ratio. The external and internal pressure are $P_{b}=-1$ and $P_{a}=1$, and the corresponding displacements are $U_{b}=-4.4232 \times 10^{-2}$ and $U_{a}=-1.3270 \times 10^{-2}$, which were obtained by solving the direct problem. The minimization of the functional $E(\tau, \eta)$ is performed by using the optimization toolbox of Matlab [43] and the state and adjoint equations have closed form solutions. The optimization process is initialized with the pair $\left(\tau_{0}=0, \eta_{0}=0\right)$ leading to an initial value of the function $E$ of $1.103 \times 10^{-2}$. Figures 4(a), 5(a) and (b) show the evolution during the optimization process of $E(\eta, \tau), \eta$ and $\tau$, respectively. Figure 4(b) shows 


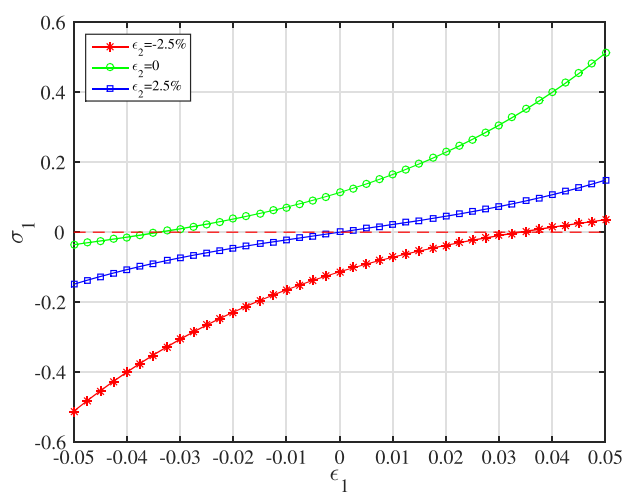

(a)

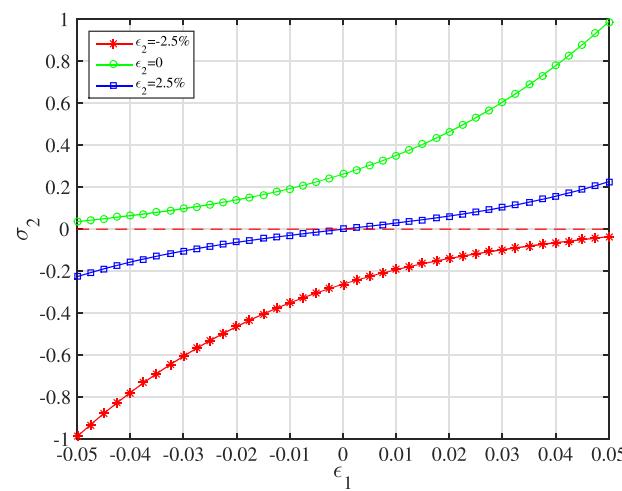

(b)

Figure 3. Stress-strain curves for the hyperelastic material.

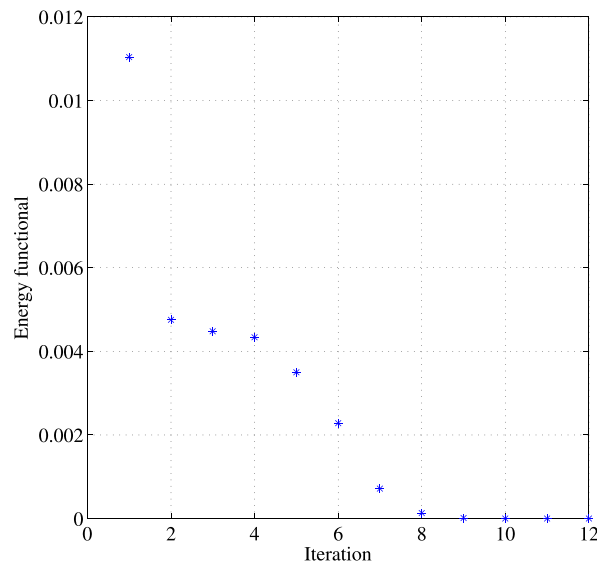

(a) Function $E(\eta, \tau)$

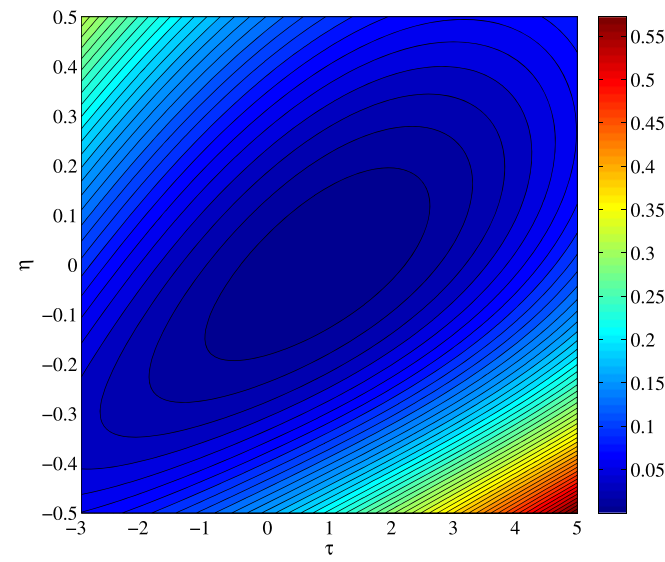

(b) Function $E(\eta, \tau)$.

Figure 4. Evolution of the function $E(\eta, \tau)$ defined by (60) during the iteration process on the left and its isolines on the right.

the isolines of the function $E(\eta, \tau)$. The function reached its minimal value after 11 iterations: $E=1.044 \times 10^{-13}$. The identified data are $U_{u}=-1.3269 \times 10^{-2}$ and $T_{u}=1$, i.e. the exact values of the direct problem.

\subsection{Elasticity with asymmetric behaviour in the traction-compression ranges}

Badel et al [44], 2007, derived a constitutive equation for geomaterials exhibiting different behaviours in traction and compression, due to the presence of micro-cracking damage. When the damage is kept constant and non-zero, the material obeys a hyperelastic law with a fixed ratio between the elastic moduli in traction and compression ranges. The $C^{1}$ hyperelastic potential $\varphi$ for an initially isotropic material is in the proposed model: 


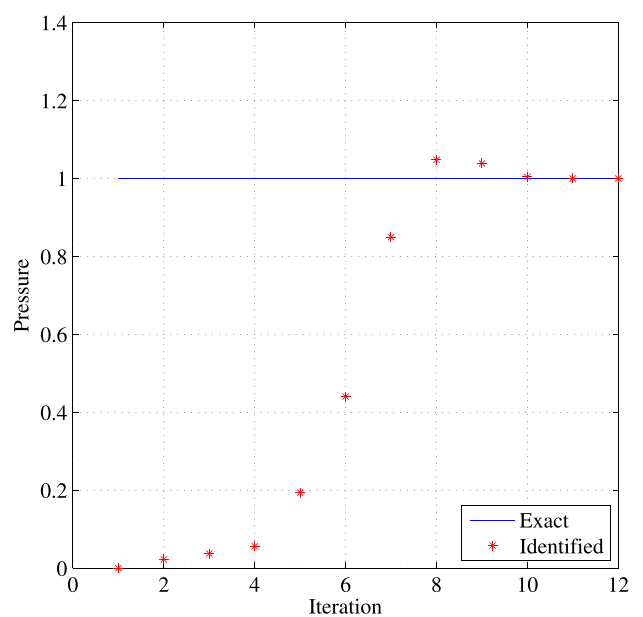

(a) Inner pressure $\eta$

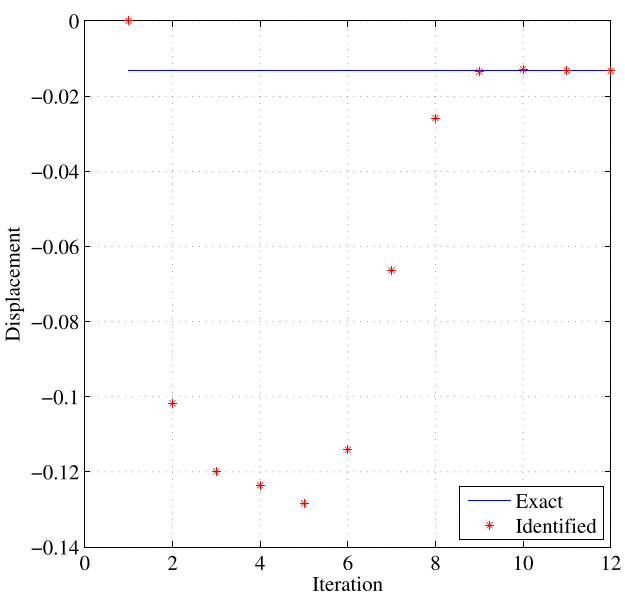

(b) Radial inner displacement $\tau$.

Figure 5. Evolution of the internal presssure $\eta$ and radial inner displacement $\tau$ during the iteration process.

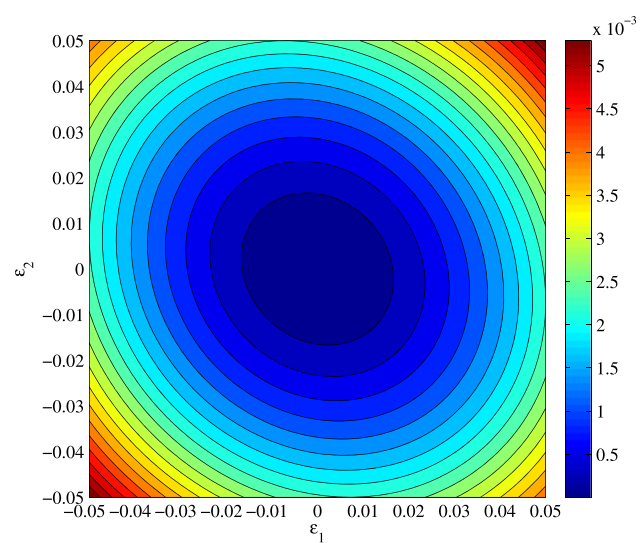

(a)

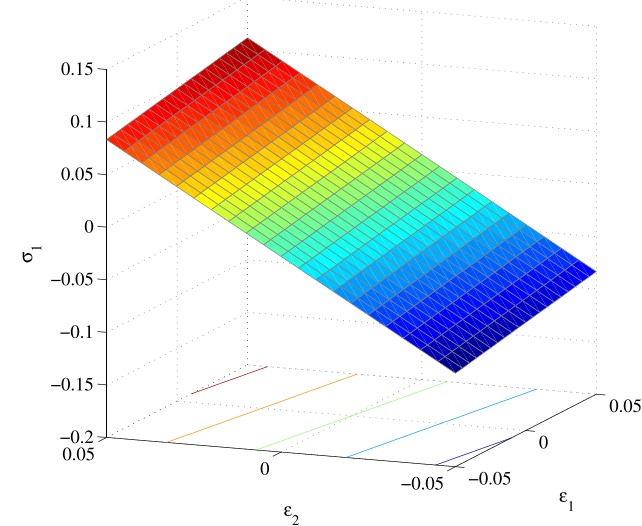

(b)

Figure 6. Isolines of the hyperelastic potential $\varphi$ in (61) and stress-strain relation for $\sigma_{1}$ in (62) as a function of $\left(\varepsilon_{1}, \varepsilon_{2}\right)$ for $\xi=1$ (linear elasticity, undamaged material).

$$
\begin{aligned}
\varphi(\varepsilon)= & \frac{\lambda}{2} \operatorname{tr}(\varepsilon)^{2}[H(-\operatorname{tr}(\varepsilon))+\xi H(\operatorname{tr}(\varepsilon))] \\
& +\mu \sum_{i=0}^{3} \varepsilon_{i}^{2}\left[H\left(-\varepsilon_{i}\right)+\xi H\left(\varepsilon_{i}\right)\right] \quad 0 \leqslant \xi \leqslant 1,
\end{aligned}
$$

where $H$ is the Heaviside function, $\varepsilon_{i}$ the eigenstrains, and $(\lambda, \mu)$ the Lamé moduli of the initially un-cracked material. The coefficient $\xi$ is lesser than one as the open cracks reduce the rigidity of the material in the traction range. The figures 6(a), 7(a) and 8(a) displays the isolines of the potential $\varphi$ in the plane of principal strains in two-dimensions, for three values of this parameter: $\xi=1$, i.e. linear elasticity, $\xi=0.3$, i.e. a ratio of 0.3 between the modulus in traction range and the modulus in compression range and $\xi=0$ corresponding to a fully 


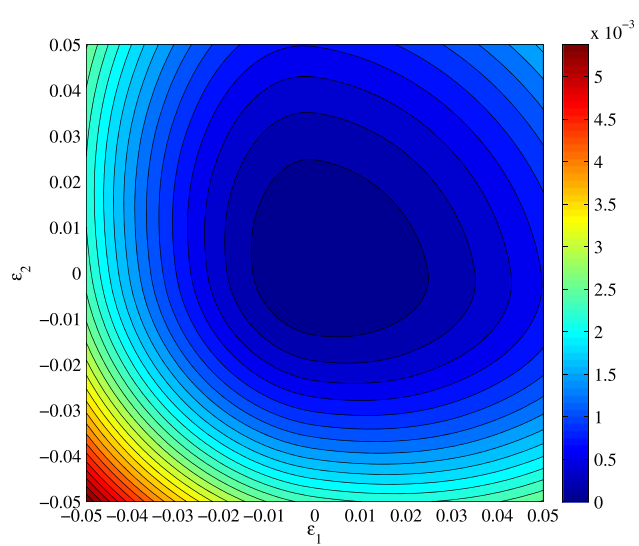

(a)

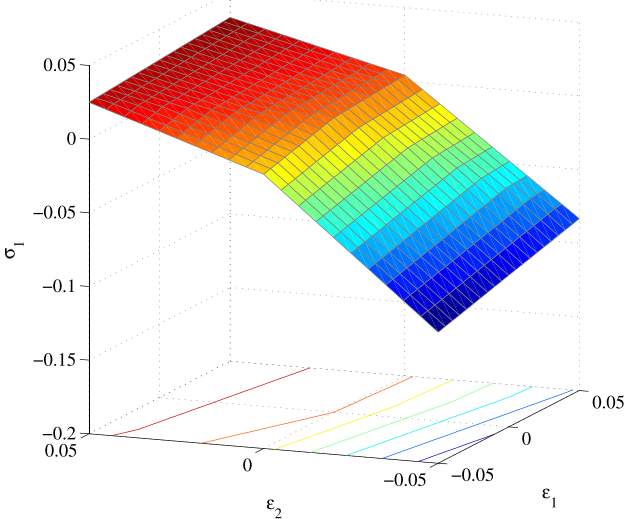

(b)

Figure 7. Isolines of the hyperelastic potential $\varphi$ in (61) and stress-strain relation for $\sigma_{1}$ in (62) as a function of $\left(\varepsilon_{1}, \varepsilon_{2}\right)$ for $\xi=0.3$.

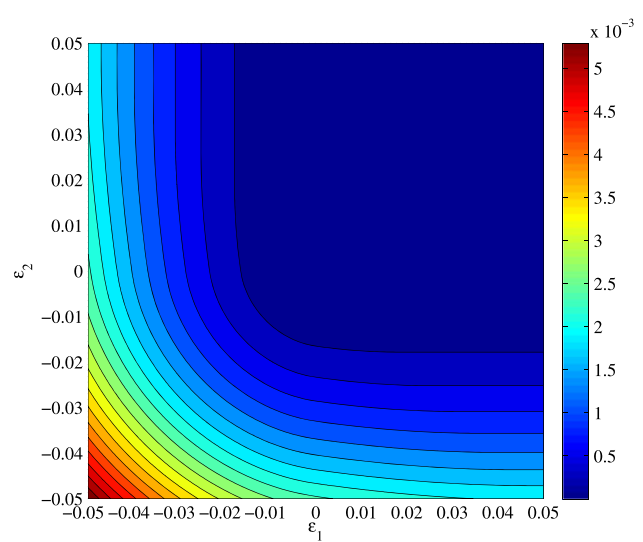

(a)

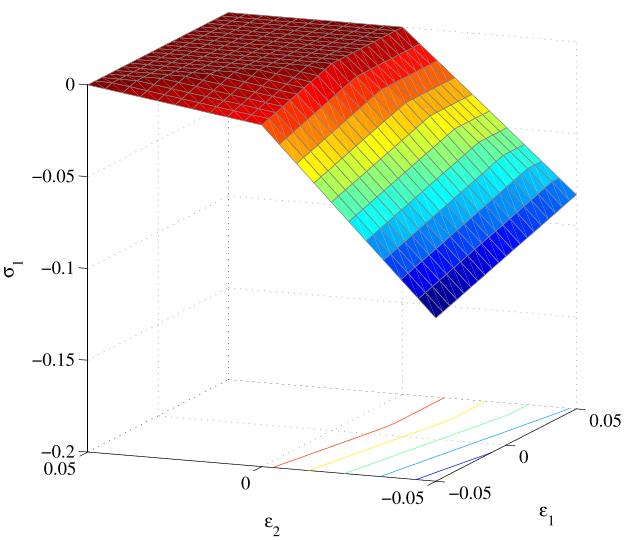

(b)

Figure 8. Isolines of the hyperelastic potential $\varphi$ in (61) and stress-strain relation for $\sigma_{1}$ in (62) as a function of $\left(\varepsilon_{1}, \varepsilon_{2}\right)$ for $\xi=0$ (fully damaged material).

damaged material in the traction range. The stress-strain relation is derived by differentiation and reads:

$$
\sigma_{i}=\lambda \operatorname{tr}(\varepsilon)[H(-\operatorname{tr}(\varepsilon))+\xi H(\operatorname{tr}(\varepsilon))]+2 \mu \varepsilon_{i}\left[H\left(-\varepsilon_{i}\right)+\xi H\left(\varepsilon_{i}\right)\right] .
$$

The stress strain law is piecewise linear because the potential is quadratic along any half-line starting from the origin in the strains space. The stress strain relation $\sigma_{1}=f\left(\varepsilon_{1}, \varepsilon_{2}\right)$ is depicted in figures 6(b), 7(b) and 8(b) for three values of the parameter $\xi: \xi=1, \xi=0$ and $\xi=0.3$. The different elasticity regimes are represented by different planes, their intersections correspond to the change of sign of the trace of the strain tensor or the change of sign of the eigenstrains, $\left(\varepsilon_{1}, \varepsilon_{2}\right)$ here.

The Cauchy problem addressed here aims at determining the traction zones in such a medium, the internal displacement and pressure on the boundary of the concrete tunnel inside 


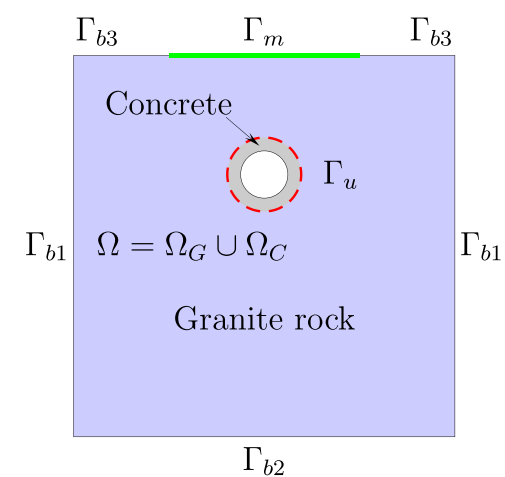

(a) Domain used in the reference problem.

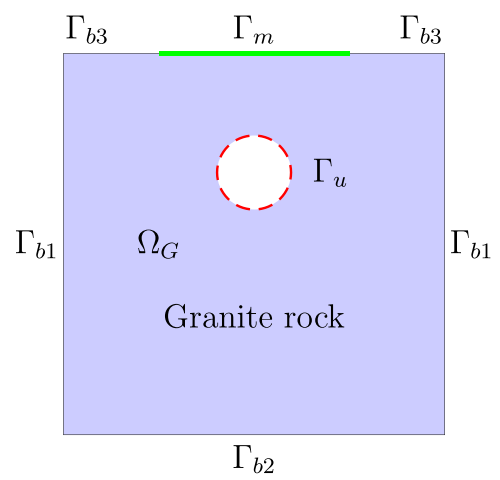

(b) Domain used in the identification problem.

Figure 9. Geometry and notations of the concrete tunnel in a qranite rock. Plane strain assumptions are considered.

it. The geometries used in the reference problem and the identification one are depicted in figures 9(a) and (b). The Cauchy data (surface pressure and surface displacement) are given on the upper boundary $\Gamma_{m}$ of the domain. $\Gamma_{u}$ the boundary where the data have to be identified is that of the cavity, more precisely the interface between the concrete and the granite rock. The software used here is CODE-ASTER ${ }^{\odot}$ which is developed by Electricité de France, that is an open source distribution. It is associated to the numerical optimization toolbox of SciPy [45]. The Cauchy data are generated by solving the forward problem defined on the reference geometry shown in the figure 9(a). A non-vanishing pressure is applied on the boundary $\Gamma_{m}$ and a vanishing pressure inside the cavity of the concrete tunnel is considered. The boundary condition are prescribed as follows: $u_{x}=0$ on $\Gamma_{b 1}, \quad u_{y}=0$ on $\Gamma_{b 2}, \quad \sigma \cdot \mathbf{n}=$ 0 on $\Gamma_{b 3} \quad \sigma \cdot \mathbf{n}=P_{m}=10^{7} \mathrm{~Pa}$ on $\Gamma_{m}$. The materials considered in this example have the following properties:

(i) Concrete (linear elastic material): Young modulus $Y_{C}=45 \mathrm{GPa}$, Poisson ratio $\nu_{C}=0.25$.

(ii) Granite: Young modulus $Y_{G}=30.0 \mathrm{GPa}$, Poisson ratio $\nu_{G}=0.2$, scalar damage parameter $d=0.3$, a material parameter governing the post-peak slope of the stressstrain curve in simple tension $\gamma=1$ so that $\xi=(1-d) /(1+\gamma d)=0.35$, see [44] and CODE-ASTER $^{\odot}$ documentation for more details.

To avoid the inverse problem crime, two different meshes were used to solve the forward problem and the inverse problem. As the potential is not twice differentiable, the adjoint problem established above cannot be used to solve the optimization problem defined by (8). Thus, we adopted the Powell algorithm where the gradient is computed by finite difference method. The convergence is obtained after 200 iterations. To test a method avoiding the computation of the gradient computation, we used the alternating direction iterative procedure to solve the optimization problem. This method is detailed in [16]. The convergence is obtained after 150000 iterations. Figures 10 and 11 depict the identified results compared to the reference ones. Figures 10 (a) and (b) show the identified displacement components $u_{x}$ and $u_{y}$ at different steps of the minimization process compared to the exact values. Figures 10(c) and (d) show the identified nodal forces $F_{x}$ and $F_{y}$ at different steps of the minimization process compared to the exact values. Figures 11(a), (c) and (e) show the identified Von Mises and principal stresses compared to the reference ones shown in figures 11(b), (d) and 


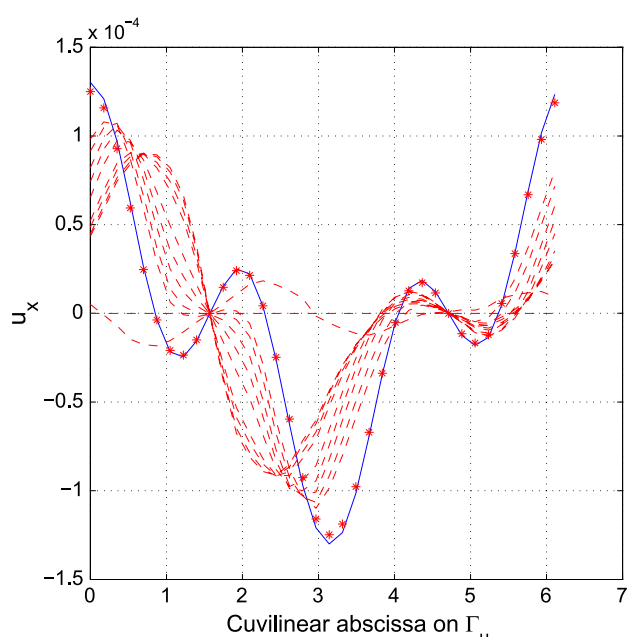

(a) Nodal displacement $u_{x}$.

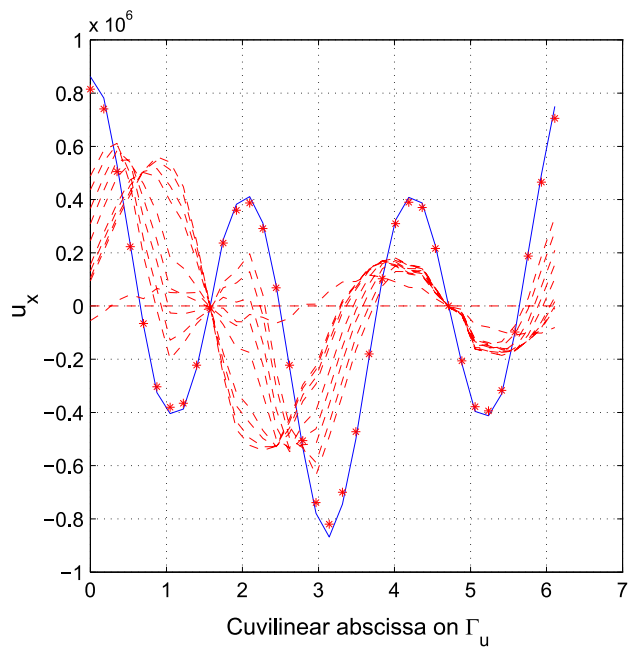

(c) Nodal load $F_{x}$

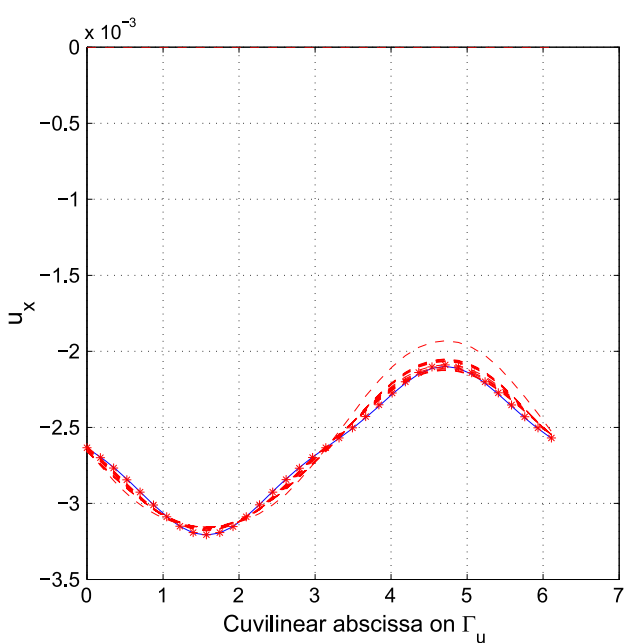

(b) Nodal displacement $u_{y}$

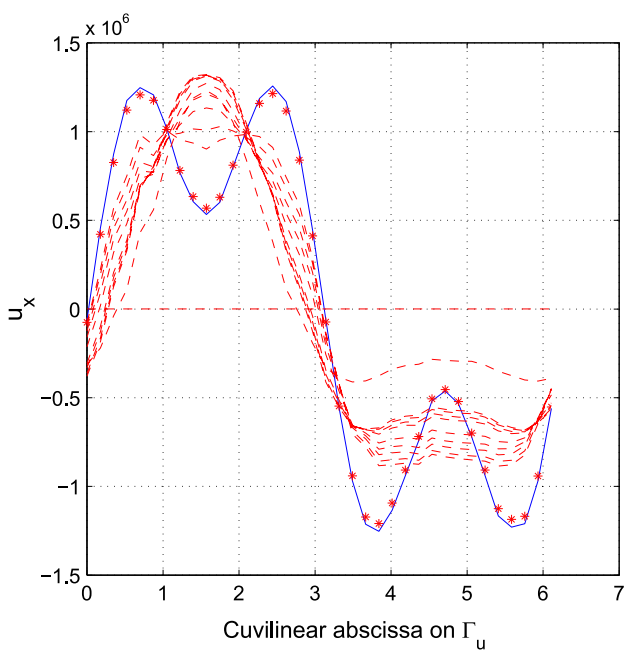

(d) Nodal load $F_{y}$.

Figure 10. Identified displacement field and nodal load on $\Gamma_{u}$ compared to the exact

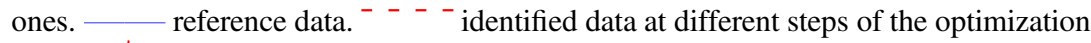
process. $*$ identified data when the optimization process converged. All initial data are taken equal to zero.

(f). We observe that all results are satisfactory: a very good precision is achieved throughout the solid up to boundaries 'far away' from the boundary where the Cauchy data are given, and the regions with various elastic regimes are perfectly identified.

\section{Conclusion}

The energy gap method for the solution of Cauchy problems or data completion problems has been extended to an error in constitutive equation method in order to deal with hyperelastic materials. The new approach encompasses the preceding addressed case of linear elasticity. 


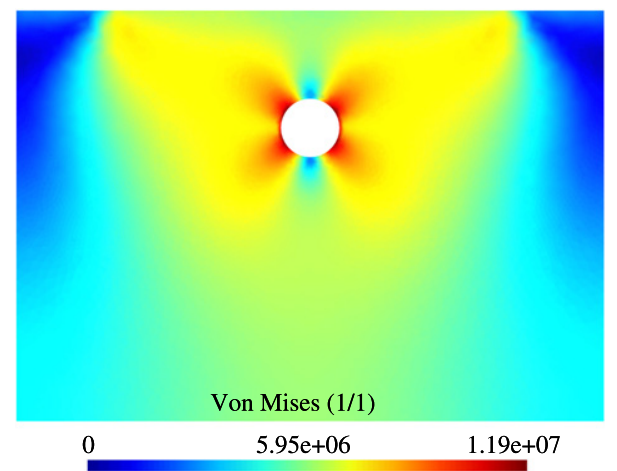

(a) Exact Von Mises stress $\sigma_{e q}$.

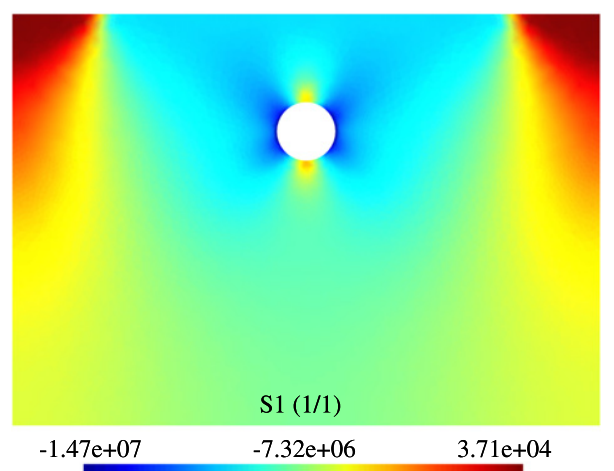

(c) Exact principal stress $\sigma_{1}$.

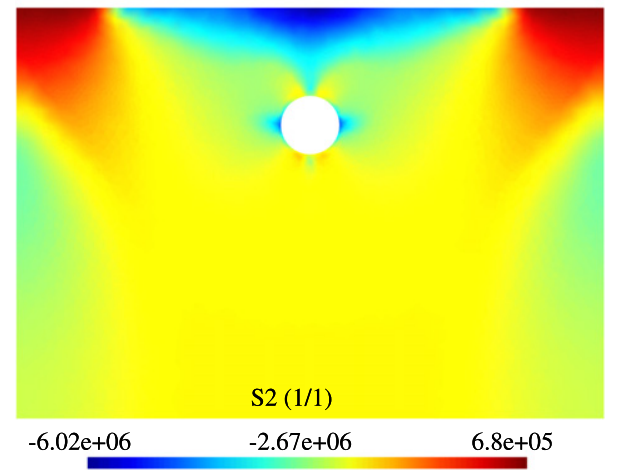

(e) Exact principal stress $\sigma_{2}$.

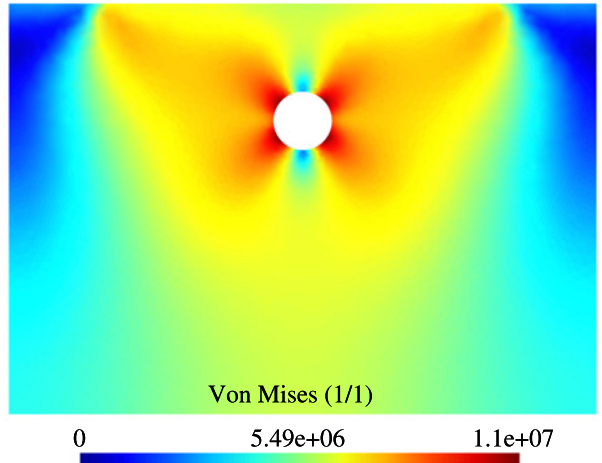

(b) Identified Von Mises stress $\sigma_{e q}$.

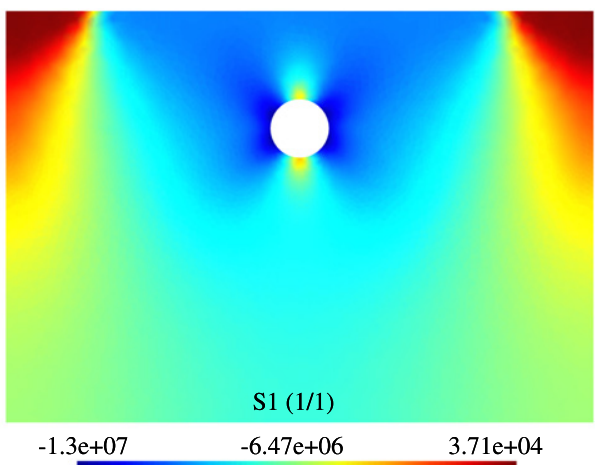

(d) Identified principal stress $\sigma_{1}$.

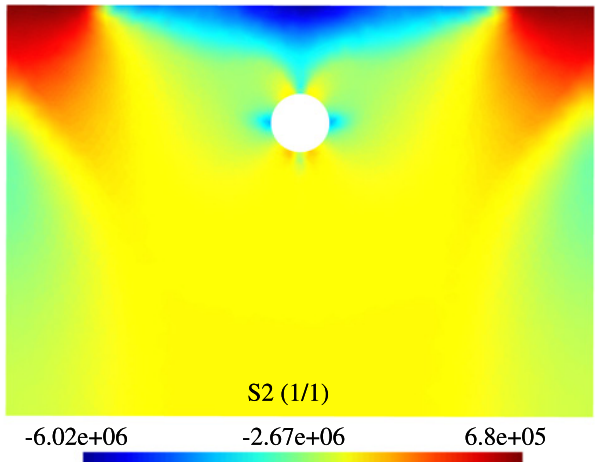

(f) Identified principal stress $\sigma_{2}$.

Figure 11. Von Mises and principal stresses distribution.

Provided the hyperelastic potential (or free energy) is lsc convex, the method proved its efficiency on two simple examples even for non-twice-differentiable potentials. In the same spirit as in the derivation of the error in constitutive equation described here, some preliminary results have been obtained for materials defined by two potentials within the elastoplasticity framework with linear isotropic hardening, see [46]. Further work will address, first, examples and applications in three-dimensions of space and the derivation of the gradient of the error functional for non-twice differentiable potentials, secondly the issues of 
regularization: up to what noise level the regularization-free method developed here is valid $[1,16,23]$ ? What kind of regularization has to be added above this noise level [47-50]?

\section{References}

[1] Andrieux S and Baranger T N 2008 An energy error-based method for the resolution of the Cauchy problem in 3D linear elasticity Comput. Methods Appl. Mech. Eng. 197 902-20

[2] Andrieux S and Baranger T N 2013 Three-dimensional recovery of stress intensity factors and energy release rates from surface full-field displacements Int. J. Solids Struct. 50 1523-37

[3] Andrieux S and Baranger T N 2012 Emerging crack front identification from tangential surface displacements C. R. Méc. 340 565-74

[4] Baranger T N and Andrieux S 2008 An optimization approach for the Cauchy problem in linear elasticity Struct. Multidiscip. Optim. 35 141-52

[5] Baranger T N and Andrieux S 2009 Data completion for linear symmetric operators as a Cauchy problem: an efficient method via energy like error minimization Vietnam J. Mech. (VAST) 31 $247-61$

[6] Hadamard J 1953 Lectures on Cauchy's Problem in Linear Partial Differential Equation (New York: Dover)

[7] Calderon A P 1958 Uniqueness in the Cauchy problem for partial differential equations Am. J. Math. 80 16-36

[8] Dehman B and Robbiano A 1993 La propriété du prolongement unique pour un système elliptique: le système de Lamé J. Math. Pure Appl. 72 475-92

[9] Fursikov A V 2000 Optimal Control of Distributed Systems (Providence, RI: American Mathematical Society)

[10] Leitão A 2000 An iterative method for solving elliptic Cauchy problems Numer. Funct. Anal. Optim. 21 715-42

[11] Kozlov V A, Maz'ya V G and Fomin A F 1992 An iterative method for solving the Cauchy problem for elliptic equations Comput. Math. Math. Phys. 31 45-52

[12] Baumeister J and Leitao A 2001 On iterative methods for solving ill-posed problems modeled by partial differential equations J. Inverse Ill-Posed Problems 9 13-29

[13] Marin L and Lesnic D 2004 The method of fundamental solutions for the cauchy problem in twodimensional linear elasticity Int. J. Solids Struct. 41 3425-38

[14] ben Belgacem F and el Fekih H 2005 On Cauchy's problem: I. A variational Steklov-Poincaré theory Inverse Problems 211915

[15] Azaïz M, ben Belgacem F and el Fekih H 2006 On Cauchy's problem: II. Completion, regularization and approximation Inverse Problems 22 1307-36

[16] Baranger T N and Andrieux S 2011 Constitutive law gap functionals for solving the Cauchy problem for linear elliptic PDE Appl. Math. Comput. 218 1970-89

[17] Cimetière A, Delvare F, Jaoua M and Pons F 2001 Solution of the Cauchy problem using iterated tikhonov regularization Inverse Problems 17 553-70

[18] Young D L, Tsai C C, Chen C W and Fan C M 2008 The method of fundamental solutions and condition number analysis for inverse problems of Laplace equation Comput. Math. Appl. 55 1189-200

[19] Marin L and Lesnic D 2002 Boundary element solution for the cauchy problem in linear elasticity using singular value decomposition Comput. Methods Appl. Mech. Eng. 191 3257-70

[20] Hon Y C and Wei T 2001 Backus-Gilbert algorithm for the Cauchy problem of the Laplace equation Inverse Problems 17261

[21] Bourgeois L 2005 A mixed formulation of quasi-reversibility to solve the Cauchy problem for Laplace's equation Inverse Problems 21 1087-104

[22] Bourgeois L 2006 Convergence rates for the quasi-reversibility method to solve the Cauchy problem for Laplace's equation Inverse Problems 22413

[23] Andrieux S, Baranger T N and Ben Abda A 2006 Solving Cauchy problems by minimizing an energy-like functional Inverse Problems 22 115-33

[24] Escriva X, Baranger T N and Tlatli Hariga N 2007 Leak identification in porous media by solving the Cauchy problem C. R. Méc. 335 401-6

[25] Klibanov M V 2015 Carleman estimates for the regularization of ill-posed Cauchy problems Appl. Numer. Math. 94 46-74 
[26] Klibanov M V 2015 A universal regularization method for ill-posed Cauchy problems for quasilinear partial differential equations arXiv: 1502.05606

[27] Baudouin L, de Buhan M and Ervedoza S 2013 Global Carleman estimates for waves and applications Commun. PDE 38 823-59

[28] Egger H and Leitao A 2008 Stable solutions of nonlinear elliptic Cauchy problems in threedimensional domains Technical Report Aachen Institute for Advanced Study in Computational Engineering Science

[29] Ekland I and Teman R 1999 Convex Analysis and Variational Problems (Philadelphia: SIAM)

[30] Bregman L M 1967 The relaxation method of finding the common point of convex sets and its application to the solution of problems in convex programming $\{$ USSR $\}$ Comput. Math. Math. Phys. 7 200-17

[31] Kiwiel K C 1997 Proximal minimization methods with generalized Bregman functions SIAM J. Control Optim. 35 1142-68

[32] Knowles I 1998 A variational algorithm for electrical impedance tomography Inverse Problems 141513

[33] Kohn R V and McKenney A 1990 Numerical implementation of a variational method for electrical impedance tomography Inverse Problems 6389

[34] Ladeveze P and Leguillon D 1983 Error estimate procedure in the finite element method and applications J. Numer. Anal. 20 485-509

[35] Ladevèze P and Chouaki A 1999 Application of a posteriori error estimation for structural model updating Inverse Problems 1549

[36] Ladeveze P 1999 Nonlinear Computational Structural Mechanics-New Approaches and NonIncremental Methods of Calculation (Berlin: Springer)

[37] Barbu V and Kunisch K 1996 Identification of nonlinear elliptic equations Appl. Math. Optim. 33 139-67

[38] Halphen B and Son Nguyen Q 1975 Sur les matériaux standard généralisés J. Mécanique 14 39-63

[39] Hadj Sassi K 2007 Une stratégie daestimation conjointe des paramètres et de laétat de structures à comportements non linéaires. Assimilation de données et erreur en relation de comportement $P h D$ Ecole Polytechnique, France

[40] Hadj Sassi K and Andrieux S 2006 Parameters identification of a nonlinear viscoelastic model via an energy error functional III European Conf. on Computational Mechanics ed C A Motasoares et al (Netherlands: Springer) p 476

[41] Chavent G 1991 On the theory and practice of nonlinear least-squares Adv. Water Resour. 14 $55-63$

[42] Griewank A 1993 Some bounds on the complexity og gradients, Jacobians and Hessians Technical Report MCS-P355-0393 Mathematics and Computer Science Division, Argonne National Laboratory

[43] MathWorks Inc 2014 MATLAB and Optimization Toolbox Release 2014b (Natick, MA: The MathWorks Inc) www.mathworks.com

[44] Badel P, Godard V and Leblond J B 2007 Application of some anisotropic damage model to the prediction of the failure of some complex industrial concrete structure Int. J. Solids Struct. 44 5848-74

[45] Jones E, Oliphant E, Peterson P et al 2011 SciPy: Open Source Scientific Tools for Python www.scipy.org (accessed 20 September 2015)

[46] Baranger T N, Andrieux S and Dang T B T 2015 The incremental Cauchy problem in elastoplasticity: general solution method and semi-analytic formulae for the pressurised hollow sphere C. R. Méc. 343 331-43

[47] Baranger T N, Johansson B T and Rischette R 2013 On the alternating method for Cauchy problems and its finite element discretisation Applied Inverse Problems (Springer Proceedings in Mathematics \& Statistics vol 48) ed L Beilina (New York: Springer) pp 183-97

[48] Rischette R, Baranger T N and Andrieux S 2013 Regularization of the noisy Cauchy problem solution approximated by an energy-like method Int. J. Numer. Methods Eng. 95 271-87

[49] Baranger T N, Andrieux S and Rischette R 2014 Combined energy method and regularization to solve the Cauchy problem for the heat equation Inverse Problems Sci. Eng. 22 1-14

[50] Rischette R, Baranger T N and Debit N 2014 Numerical analysis of an energy-like minimization method to solve a parabolic Cauchy problem with noisy data J. Comput. Appl. Math. 271 $206-22$ 